\title{
Experimental observation of dynamic ductile damage development under various triaxiality conditions - description of the principle
}

\author{
L. Pillon \\ CEA, DAM, DIF, Bruyères-le-Châtel, 91297 Arpajon Cedex, France
}

\begin{abstract}
The Gurson model has been extended by Perrin to describe damage evolution in ductile viscoplastic materials. The so-called Gurson-Perrin model allows representing damage development with respect to strain-rate conditions. In order to fill a lack in current experimental procedures, we propose an experimental project able to test and validate the Gurson-Perrin model under various dynamic conditions and for different stress triaxiality levels.
\end{abstract}

\section{Introduction}

Ductile fracture finds its origin in microscopic mechanisms: the nucleation of voids that grow and coalesce in order to form a crack. A large part of literature deals with the development of models predicting each stage of this complex phenomenon. One of the most modern theories, the micro-mechanics, links microscopic phenomena and their macroscopic consequences. The most popular of these models, proposed by Gurson [1], aims at describing the plastic behaviour of a porous ductile material by expressing its flow potential. The major interest of this model is its ability to consider the effect of stress triaxiality $\tau=\Sigma_{\mathrm{m}} / \Sigma_{\text {eq }}$, defined as the ratio of the hydrostatic stress $\Sigma_{\mathrm{m}}$ and the equivalent Von-Mises stress $\Sigma_{\text {eq }}$, on damage evolution. Numerous studies have actually shown that it was a crucial parameter for damage prediction [2,3].

Although the Gurson model has been a major advance in damage description, it is based on restricting assumptions. Extensions of the original model are necessary to provide reasonnable comparisons to experiments. In one of these extended forms, the so-called Gurson-Perrin model includes viscoplastic effects, which are essential to describe damage at high strain-rates [4]. In the Gurson-Perrin model, material parameters have to be identified from suitable experiments. Nevertheless, it will be shown in the following document that classical experimental procedures are not appropriate to determine these parameters. In the first part of this document, we describe the Gurson-Perrin model and equations allowing a dynamic description of damage evolution. We point out the limits of current experimental techniques. In the second part, we describe a new experimental procedure that will be tested soon. First numerical predictions are described.

\section{The Gurson-Perrin model}

The Gurson model has been extended by Perrin to describe the viscoplastic behaviour of a ductile porous material [4]. Like the Gurson one, the Gurson-Perrin model is a micromechanical model that is based on assumptions on the matrix behaviour, at the microscopic scale. The matrix material is supposed to follow a Bingham law associated with a Von-Mises plasticity criterion:

$$
\sigma_{\mathrm{eq}}=Y_{0}+3 \eta_{0} d_{\mathrm{eq}}^{\mathrm{p}}
$$

with $\sigma_{\mathrm{eq}}=\sqrt{\sigma^{\prime}: \sigma^{\prime}}$ the equivalent Von-Mises stress, $d_{\mathrm{eq}}^{\mathrm{p}}=\sqrt{\mathbf{d}^{\mathrm{p}^{\prime}}: \mathbf{d}^{\mathrm{p}^{\prime}}}$ the equivalent Von-Mises plastic rate of deformation, $\sigma^{\prime}$ and $\mathbf{d}^{\mathrm{p}^{\prime}}$, the deviatoric part of stress tensor and plastic rate of deformation tensor and $Y_{0}$ and $\eta_{0}$ the matrix elastic limit and viscosity parameter. Lowercase letters refer to the matrix material, while capital letters concern the porous material.

The matrix material forms a hollow sphere, for which the rate of void is called the porosity, noted $f$. Mechanical characteristics of this hollow sphere are representative of the macroscopic properties of a Representative Elementary Volume (REV) of porous material with the same porosity $f$. From the assumptions at microscale and the geometry of the REV, Perrin exhibits the viscoplastic potential of the porous material:

$$
\begin{gathered}
\Sigma_{\mathrm{m}}=\underbrace{3 \zeta(f) D_{\mathrm{m}}^{\mathrm{p}}}_{\text {Viscous stress }}+2 Y_{0} / 3[\operatorname{arcsh}(\lambda / f)-\operatorname{arcsh} \lambda] \\
\Sigma_{\text {eq }}=\underbrace{3 \eta(f) D_{\mathrm{eq}}^{\mathrm{p}}}_{\text {Viscous stress }}+Y_{0}\left[\sqrt{1+\lambda^{2}}-\sqrt{f^{2}+\lambda^{2}}\right]
\end{gathered}
$$

with the triaxiality of the plastic rate of deformation tensor $\lambda=2 D_{\mathrm{m}}^{\mathrm{p}} / D_{\mathrm{eq}}^{\mathrm{p}}$ and the two porous viscosity parameters $\eta(f)=(1-f) \eta_{0}$ and $\zeta(f)=4(1-f) \eta_{0} /(3 f)$. Equations (2) and (3) differ from the Gurson's ones by the addition of hydrostatic and equivalent viscous stresses. This model reduces to the Gurson plasticity model for $\eta_{0}=0$.

A second property has to be pointed out:

$$
\begin{aligned}
& \Sigma_{\mathrm{m}}(\lambda, f \rightarrow 0) \rightarrow \infty \\
& \Sigma_{\mathrm{eq}}(\lambda, f \rightarrow 0)=Y_{0}\left[\sqrt{1+\lambda^{2}}-|\lambda|\right]+3 \eta_{0} D_{\mathrm{eq}}^{\mathrm{p}}
\end{aligned}
$$

Consequently, the model cannot be used for ductile materials with low porosity. This behaviour already appeared in the Gurson model. This variable has to be restricted to a minimum $f=f_{0}$, with $f_{0}$ the initial porosity, a material parameter. Starting with this initial porosity, the evolution of voids in the material results from the growth 
of existing voids. The rate of increase is obtained by the plastic incompressibility of the matrix:

$$
\dot{f}=3(1-f) D_{\mathrm{m}}^{\mathrm{p}}
$$

An accelerated porosity model $f^{*}(f)$ can be used to account for effects of rapid void coalescence on mechanical properties [5]. It can be introduced in viscoplastic equations (2) and (3) in place of the porosity $f$. Under a critical porosity, $f^{*}(f) \approx f$ and above this critical value, $f^{*}(f)>f$ and $f^{*}(f)$ evolves faster than $f$ until it reaches its maximum $f^{*}(f)=f_{\max }$.

Concerning the elastic part of the description, the porous shear modulus proposed by MacKenzie is used for the deviatoric part of the stress tensor [6]. The hydrostatic part of the stress tensor $\Sigma_{\mathrm{m}}$, defined as the opposite of the hydrostatic pressure $P$, results from a porous Equation of State, which is compatible with a description at high strainrates (impact loadings ....).

This full description of damage evolution associated with the Gurson-Perrin model is well-adapted to high strain-rates. Some parameters like $Y_{0}, \eta_{0}, f_{0}$ have to be identified with suitable dynamic experiments. For the Gurson model, parameters are usually identified from simple tensile tests on notched bars. This experimental concept is actually well-adapted to the parameters identification of the Gurson model since the level of stress triaxiality can be controlled by the geometry of the notch. Consequently, a change in the notch size induces a change in the amount of stress triaxiality that controls the evolution of damage. Thus, the time at which each specimen breaks, depends on the triaxiality and on the geometry of the notch. This experimental procedure can be performed more dynamically on Hopkinson bars. Strain-rates reach $10^{4} \mathrm{~s}^{-1}$, which is weaker than in impact loadings $\left(10^{6} \mathrm{~s}^{-1}\right)$. This second type of experiment is often used in order to fix dynamically the parameters of the Gurson model. Nevertheless, this experimental procedure is not adapted to the validation of a Gurson-type model, since damage evolves with pressure, not with triaxiality.

In the next paragraph, we present an experimental project that allows to test the Gurson-Perrin model under dynamic conditions and for different triaxiality levels.

\section{Experimental procedure and numerical predictions}

The configuration presented here is a prototype. The good behaviour of this experimental concept will be checked soon. Consequently, a few parameters like dimensions and velocities can be slightly changed if necessary.

The new test method proposed here combines two experimental principles: plate impact experiments and tensile tests on notched bars. A schematic for the general geometry is provided in figure 1 .

Like in a flyer-plate experiment, a projectile assembly composed of a plate in aluminium and an impacting plate in copper is impacted on a target in copper with an initial velocity $\mathrm{V}_{\mathrm{i}}=400 \mathrm{~m} \cdot \mathrm{s}^{-1}$. The aluminium and impacting plate diameters are $60 \mathrm{~mm}$ and $50 \mathrm{~mm}$, while the thickness

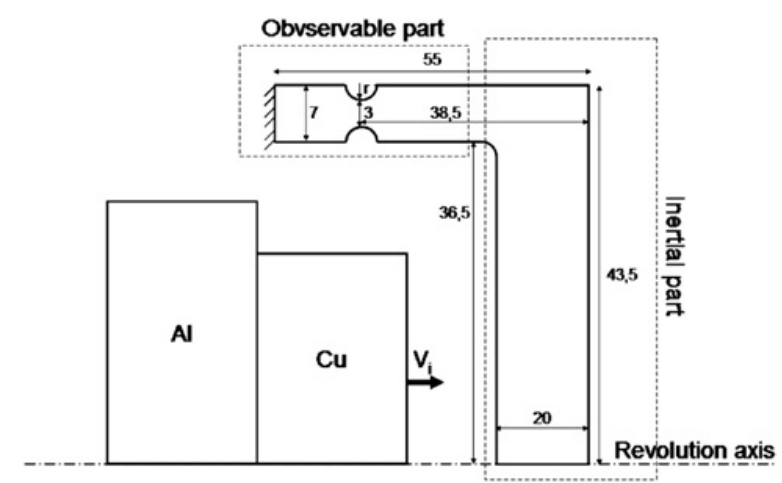

Fig. 1. Geometry of the experiment. The assembly is axisymetric (dimensions are in $\mathrm{mm}$ ).

Table 1.

\begin{tabular}{|c|c|c|c|}
\hline & Geometry 1 & Geometry 2 & Geometry 3 \\
\hline $\begin{array}{c}\text { Radius of } \\
\text { the notch }(\mathrm{mm})\end{array}$ & 1,5 & 2 & 4 \\
\hline $\begin{array}{c}\text { Fracture time } \\
(\mu \mathrm{s})\end{array}$ & 85 & 90 & 100 \\
\hline
\end{tabular}

of the copper plate is $10 \mathrm{~mm}$. This two projected plates are stuck together. By impacting the target, the impacting plate causes the acceleration of a part of the target, called the 'inertial part'. The main function of the inertial part is to generate a stable and durable tensile state in a second part of the target called the 'observable part', where the damage phenomenon will be observed. After the motion of the inertial part, this tensile state quickly appears in the observable part since the other extremity of the target is embedded. The observable part is a notched cylinder. By creating a tensile state in the observable part, a triaxiality level appears in the notched geometry, which leads to a local and gradual increase of the porosity. The inertial part generates this tensile state in the observable part until the specimen breaks.

Damage in the notched cylinder grows with the same mechanism as in tensile tests. The main difference lies in the way to create a tensile state in the observable part by using the flyer impact principle. The goal of this method is to reach higher strain-rates than in Hopkinson bars, which is possible by increasing the initial velocity. Three notch geometries are proposed in order to test different levels of stress triaxiality. Notch dimensions for each experiment are provided in the Table 1.

Stress triaxiality predictions at the centre of the notched cylinder and evolution of the porosity at this site are plotted in figures 2 and 3. The variation of the notch geometry induces a variation of stress triaxiality and different evolutions of porosity. The mechanism is the same as the one observed in tensile tests of notched bars. Each experiment ends when the target is broken; a first evaluation of the duration of each experiment is given in Table 1. Since the variation of the notch geometry induces a variation of stress triaxiality, fracture times are different for each geometry, as expected too.

Different diagnostic ways are used during the experiment in order to observe the deformation of the specimen. At first, an observation of displacements will be performed 


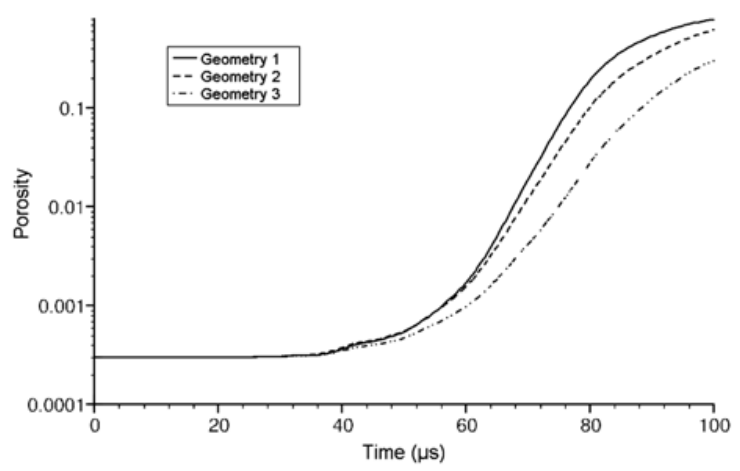

Fig. 2. Evolution of porostiy at the centre of the notched cylinder for each geometry.

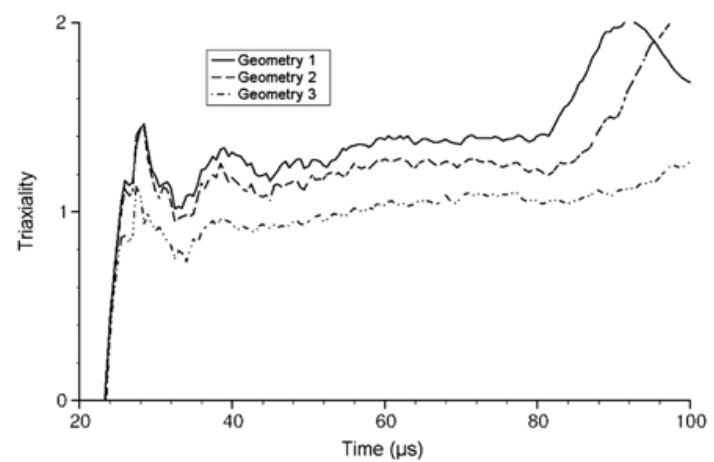

Fig. 3. Evolution of stress triaxiality at the centre of the notched cylinder for each geometry.

by heterodyne velocimetry (HV) at different points located around the specimen. Displacements in the inertial part of the specimen will be measured at four different places, located at $\mathrm{r}=0 \mathrm{~mm}, \mathrm{r}=20 \mathrm{~mm}, \mathrm{r}=38 \mathrm{~mm}$ and $\mathrm{r}=$ $42 \mathrm{~mm}$. By the same way, displacements in the observable part will be recorded at three places: one in the notch, to measure the local deformation and two outside the notch to measure the small possible rotation of the specimen. A second type of observation of the observable part will be performed during the experiment with a numeric camera. The local shape of the specimen will be precisely observed. The quality of the embedding will be checked by a deformation gauge placed between the notch and the embedded extremity. At the end of the experiment, the specimen will be broken into two parts. Consequently, a micrographic analysis of the fracture zone has been requested.
The precise conception of the overall geometry and the diagnostics are currently in progress.

\section{Conclusion}

A viscoplastic description of ductile damage is obtained by an extension of the Gurson model, called the GursonPerrin model. This modelling allows to simulate damage as well at low strain-rates as at high strain-rates. Nevertheless, a new experimental procedure is necessary to provide a good adjustment of free parameters of this model. The purpose of the experimental project presented here is to perform a damage experiment under dynamic conditions and for different triaxiality levels.

We propose a set of dynamic experiments, in which damage evolution depends on triaxiality. This experimental concept combines principle of impact loadings and tensile tests of notched bars. A change in the shape of the specimen allows an evolution of the stress triaxiality. During the experiment, the evolution of the specimen deformation is observed by $\mathrm{HV}$ measurements and by numeric camera observation. Post-mortem micrographic analysis allows a comparison between predictions and experimental observations.

\section{Acknowledgements}

The author gratefully acknowledges Franck Dambakizi for his work on the numerical implementation of the Gurson-Perrin model and Lise-Marie Adolf for the conception of the experiment.

\section{References}

1. A. Gurson, J. Engng. Mater. Technol., 99, 2 (1977)

2. A. Needleman, V. Tvergaard, J. Mech. Phys. Solids, 32, 461 (1984)

3. R. Becker, A. Needleman, O. Richmond, V. Tvergaard, J. Mech. Phys. Solids, 36, 317 (1988)

4. G. Perrin, J. Engng. Mech, 128, 885 (2002)

5. V. Tvergaard, Ad. in Ap. Mech, 27, 83 (1989)

6. J. Mackenzie, Proc. Phys. Soc. B, 63, 2 (1950) 